\title{
Spontaneous Regression of Cervical Disc Herniation: A Case Report
}

\author{
Seong Rok Han, Chan Young Choi \\ Department of Neurosurgery, Ilsan Paik Hospital, Inje University College of Medicine, Goyang, Korea
}

Spontaneous regression of cervical disc herniation is a rare, and such reports are few. A 39 year-old woman complained of severe neck pain associated with tingling and numbness of right upper extremity. The MRI of the cervical spine revealed a posterior disc extrusion at the C4-C5 level in the right para-central location. The patient was treated with conservative management without any surgical treatment. The patient's symptoms were significant improvement. After two years later, we performed follow-up cervical MRI that revealed significant spontaneous regression of the C4-C5 intervertebral disc extrusion.

Key Words: Cervical $\cdot$ Disc $\cdot$ Herniation $\cdot$ Regression

\section{INTRODUCTION}

Spontaneous regression of herniated lumbar disc has been has been well established ${ }^{3,7)}$. However, spontaneous regression of herniated cervical disc is rarely reported ${ }^{3,7,8)}$. Recent advances in magnetic resonance imaging (MRI) have facilitated followup of intervertebral disc herniation and prospect spontaneous regression ${ }^{1,8)}$. We present a case of spontaneous regression of cervical disc herniation who treated conservative management without any surgical treatment and review related literatures.

\section{CASE REPORT}

A 39 year-old woman presented with severe neck pain associated with tingling sensation and numbness of right upper extremity that developed 3 months ago. Her neurologic examinations revealed no motor weakness but hypoesthesia right C4 and C5 dermatome. All reflex brisk and symmetric. The plain radiographs of the cervical spine revealed no abnormality. The MRI of the cervical spine was done, which showed a posterior disc extrusion at the C4-C5 level in the right paracentral location (Fig. 1). Conservative management was given in the form of non-steroidal-anti-inflammatory drugs and a

- Received: August 27, 2014 • Revised: October 19, 2014

- Accepted: October 21, 2014

Corresponding Author: Seong Rok Han, MD, PhD

Department of Neurosurgery, Ilsan Paik Hospital, Inje University, 170

Juhwa-ro, Ilsan-Seo-gu, Goyang 411-410, Korea

Tel: +82-31-910-7730, Fax: +82-31-915-0885

E-mail: hsrkmj@paik.ac.kr

ÐThis is an Open Access article distributed under the terms of the Creative

Commons Attribution Non-Commercial License (http://creativecommons.org/ licenses/by-nc/3.0/) which permits unrestricted non-commercial use, distribution, and reproduction in any medium, provided the original work is properly cited. muscle relaxant. The patient also received multiple sessions of physical therapy. After 3 weeks treatment, the patient's symptoms were significant improvement. After two years later, she re-visited our hospital because of developed neck pain recently. We performed follow-up cervical MRI that revealed significant spontaneous regression of the C4-C5 intervertebral disc extrusion (Fig. 2). The patient's symptoms were alleviated spontaneously after 2 weeks.

\section{DISCUSSION}

The mechanism of spontaneous regression disc herniation is still unclear. Many factors related to the resorption process have been recognized ${ }^{1)}$. The type of disc herniation is more contributable to spontaneous disc regression than the size of disc herniation ${ }^{6}$. Spontaneous regression of herniated disc was seen more frequently in extruded disc than protruded $\operatorname{disc}^{7}$. In general, large-sized herniated disc have been observed to regress more than smaller one $e^{4,8)}$, and a sequestrated-type disc herniation is likely to regress more readily than a protruded disc $^{8)}$. Extrusion and sequestrated disc into the epidural space induces an inflammatory cascade involving neovascularization and phagocytosis that may lead to disc regression ${ }^{5,6,8)}$.

Burke et al. ${ }^{2}$ reported that the human disc tissue is capable of spontaneously producing the pro-inflammatory chemokines monocyte chemo-attractant such as protein-1 and interleukin-8. The chemo-attractants are suspected of initiating an inflammatory recruitment of monocytes from the nearby blood supply. Chemotactic for macrophages and capillaries may explain the ingrowth of granulation tissue seen in spontaneous disc herniation resorption.

Autio et al. ${ }^{1)}$ reported that a gadolinium-diethylenetriamine pentaacetic acid (Gd-DTPA) enhanced MRI to evaluate the 

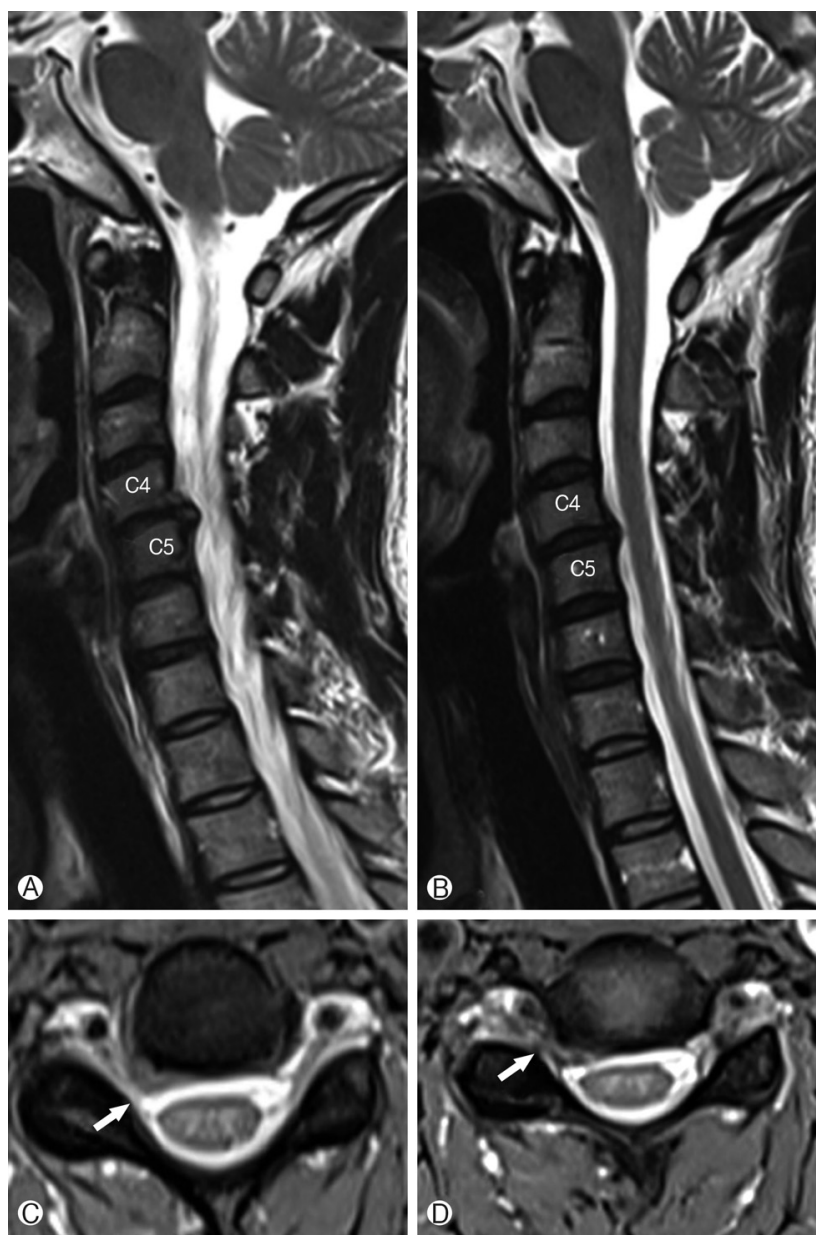

Fig. 1. Initial MRI study (T2-weighted image) of the cervical spine (A, parasagittal; B, mid-sagittal; C, D, axial) revealed a large right posterolateral extruded disc (arrow) at the C4-5 level compressing the right $\mathrm{C} 5$ root.

neovascularization zone and to predict the possibility of spontaneous regression of herniated disc. The extent of peripheral enhancement tends to be greater in sequestration type herniation when compared to bulging or protruding herniation ${ }^{4)}$.

They suggested that neovascularization in the outermost areas of herniated disc, presenting as an enhancing rim in Gd-DTPA MRI, is thought to be a major determinant of spontaneous regression of herniated disc ${ }^{1)}$ and thickness of rim enhancement was a stronger determinant of spontaneous regression than extent of rim enhancement also ${ }^{1)}$.

Kobayashi et al..$^{6}$ reported a case of herniated cervical disc that showed partial spontaneous regression on follow-up MRI after 3 weeks and complete spontaneous regression on followup MRI after 12 months. They also reviewed previous reported 6 cases of spontaneous regression in herniated cervical disc. In their report, spontaneous regression took 2 to 28 months. Mean age was 39.8 (ranged from 31 to 50). All patients sho-
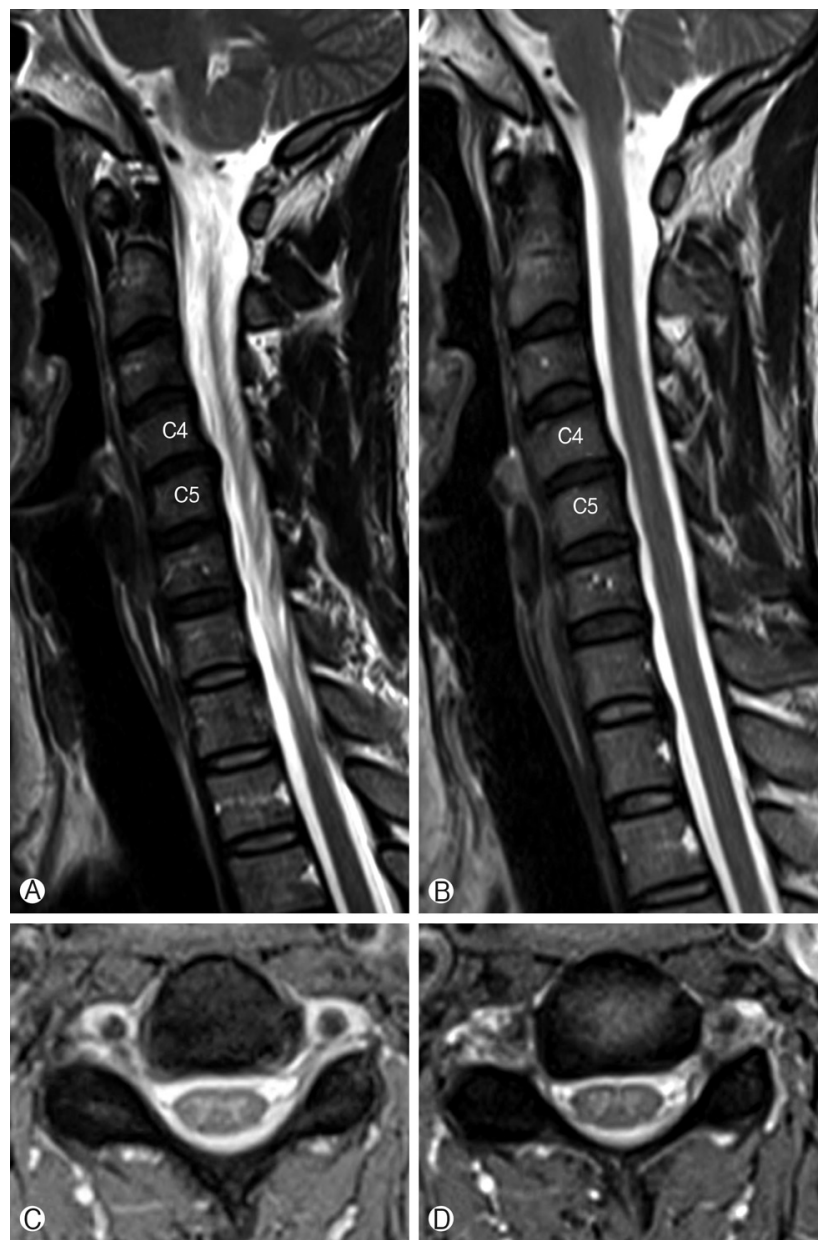

Fig. 2. After 2 years, follow-up MRI study (T2-weighted image) of the cervical spine (A, parasagittal; B, mid-sagittal; C, D, axial) showed complete spontaneous regression of the extrude disc at the C4-5 level without right C5 root compression.

wed extruded herniated disc type. Our case is similar with these reported cases.

\section{CONCLUSION}

Spontaneous regression of herniated cervical disc is rare. However, some cervical disc herniation that are likely to regress spontaneously for very specific and predictable reasons. In some cases, conservative management should be considered an option for treatment.

\section{REFERENCES}

1. Autio RA, Karppinen J, Niinimäki J, Ojala R, Kurunlahti M, Haapea $\mathrm{M}$, et al: Determinants of spontaneous resorption of intervertebral disc herniations. Spine (Phila Pa 1976) 31(11): 
1247-1252, 2006

2. Burke JG, Watson RW, McCormack D, Dowling FE, Walsh MG, Fitzpatrick JM: Spontaneous production of monocyte chemoattractant protein-1 and interleukin- 8 by the human lumbar intervertebral disc. Spine (Phila Pa 1976) 27(13):1402-1407, 2002

3. Gurkanlar D, Yucel E, Er U, Keskil S: Spontaneous regression of cervical disc herniations. Minim Invasive Neurosurg 49(3): 179-183, 2006

4. Kim ES, Oladunjoye AO, Li JA, Kim KD: Spontaneous regression of herniated lumbar discs. J Clin Neurosci 21(6):909-913, 2014

5. Kim KD, Kim ES: Unknown case: part 2. Spontaneous regression of herniated disc. Spine (Phila Pa 1976) 38(4):368, 2013

6. Kim SG, Yang JC, Kim TW, Park KH: Spontaneous regression of extruded lumbar disc herniation: three cases report. Korean J Spine 10(2):78-81, 2013

7. Kobayashi N, Asamoto S, Doi H, Ikeda Y, Matusmoto K: Spontaneous regression of herniated cervical disc. Spine J 3(2):171-173, 2003

8. Orief T1, Orz Y, Attia W, Almusrea K: Spontaneous resorption of sequestrated intervertebral disc herniation. World Neurosurg 77(1):146-152, 2012 